\title{
Detection of Arsenic Dopant Atoms in Silicon Crystal by Aberration Corrected Scanning Transmission Electron Microscopy
}

\author{
Y. Oshima***, Y. Hashimoto**, H. Sawada****, N. Hshikawa****, K. Asayama****, Y. \\ Kondo $* * * *$ and $\mathrm{Y}$. Tanishiro $* * * * * *, \mathrm{~K}$. Takayanagi $* * * * * *$ \\ * JST, CREST, 5-Sanbancho, Chiyoda-ku, Tokyo, 102-0075, Japan \\ ** Dep. Mat. \& Sci. Eng., Tokyo Tech., J1-3, 4259 Nagatsuta, Midori-ku, Yokohama, 226-8502, \\ Japan. \\ *** JEOL Ltd., 3-1-2 Musashino, Akishima, Tokyo 196-8558, Japan \\ **** Renesas Technology Corp., 5-20-1 Josuihon-cho, Kodaira, Tokyo 187-8588, Japan. \\ ***** Dep. Phys., Tokyo Tech., 2-12-1-H-51 Oh-okayama, Meguro-ku, Tokyo 152-8551, Japan
}

The gate width of an integrated circuit has been downsized to be a few tens $\mathrm{nm}$ in silicon devices. In such a narrow gate, it has been reported that source-drain current is not controlled by the gate bias voltage and the current has been suggested to be influenced by the dopant fluctuation. In order to clarify this suggestion, it is necessary to confirm the distribution of dopant atoms. Voyles et al. has successfully demonstrated imaging of individual antimony ( $\mathrm{Sb}$ ) dopant atoms or clusters in highly $\mathrm{n}$ type silicon by scanning transmission electron microscopy (STEM) [1]. But, arsenic (As) atoms as commonly dopant elements has not been detected clearly [2]. In this study, we observed individual arsenic (As) dopant atoms using our newly developed aberration corrected STEM (R005) [3].

Silicon wafer was doped with arsenic (As) ions implanted at $30 \mathrm{keV}$ and annealed by rapid thermal treatment. The concentration was analyzed to be $5 \times 10^{20} \mathrm{~cm}^{-3}$ around $30 \mathrm{~nm}$ depth from the surface, measured by secondary ion mass spectroscopy (SIMS). The STEM specimen was carefully prepared by mechanical polishing, dimpling and low angle ion milling. In this study, we have done high-angle annular dark-field STEM (HAADF-STEM) observation in the $<001>$ zone-axis orientation to detect arsenic (As) dopant atoms in Si crystal. The observed regions were about $10 \mathrm{~nm}$ in thickness, measured by electron energy loss spectroscopy.

Fig. 1 shows a typical HAADF-STEM image of a region ( $4 \mathrm{~nm} \times 4 \mathrm{~nm}$ ) containing As atoms. Among about 460 spots corresponding to atomic columns along the $<001>$ direction, we found about 20 brighter spots. The intensity ratio of the brighter spots to the average of the others was above 1.3. We investigated the intensity ratio of As doped atomic column to non-doped one using STEM image simulation. Fig. 2 shows the defocus dependence of the ratio. An As dopant atom is assumed to be set in the middle of $10 \mathrm{~nm}$ thickness. We find that the intensity ratio drastically increases when the in-focus plane is close to the position of the As dopant atom, and is above 1.3 when the in-focus plane is within the thickness range between 4 and $7 \mathrm{~nm}$. Therefore, an atomic column, which includes an As dopant atom located within the slice of $3 \mathrm{~nm}$ thickness around the infocus plane of the STEM probe, can be imaged 1.3 times brighter than the non-doped Si columns. Assuming that the concentration of As dopant atoms is $0.8 \%$, the number of As dopant atoms within the slice of $3 \mathrm{~nm}$ thickness is estimated to be about 20 following the average density of the present specimen. The concentration of $0.8 \%$ is comparable to the results obtained by SIMS. Therefore, the number and intensity of the brighter spots in the HAADF-STEM image of Fig.1 are well explained by As dopant atoms located within the slice of $3 \mathrm{~nm}$ thickness around the in-focus plane of the 
STEM probe. We conclude that individual As dopant atoms in Si crystal are detected using our developed STEM [3].

[1] P. M. Voyles, D. A. Muller et al., Nature 416 (2002) 826.

[2] T. Yamazaki et al., Phys. Rev. B 61 (2000) 13833.

[3] H. Sawada et al., Jpn. J. Appl. Phys. 46 (2007) L568. and H. Sawada et al., Microsc.

Microanal.14 (2008) 802.

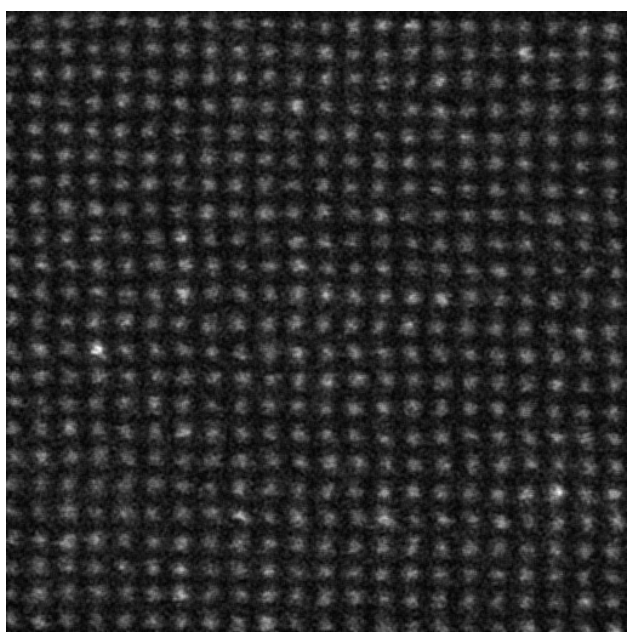

Fig. 1 A typical HAADF-STEM image of arsenic (As) dopant atoms in silicon crystal. This image is taken at $300 \mathrm{keV}$, $\mathrm{Cs}=0$, and the half-convergent beam of 30 $\mathrm{mrad}$, and by collecting the electrons scattered at angles between approximately 60 and $160 \mathrm{mrad}$. This image is processed by area averaging ( $3 \times 3$ pixels) and subtracting background using rolling ball method. Note that length per pixel is $8 \mathrm{pm}$, while the distance between two neighboring spots is $192 \mathrm{pm}$.

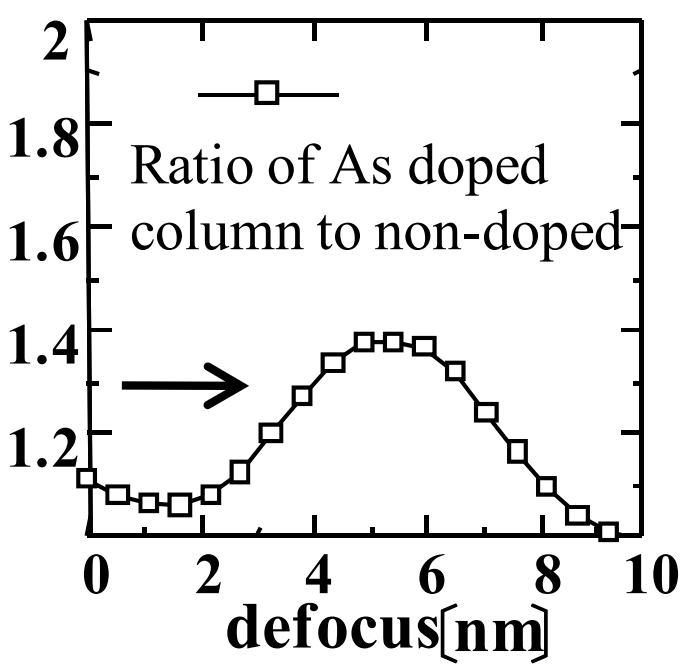

Fig. 2 Defocus dependence of the intensity ratio, which is determined to be the ratio of the intensity of As doped atomic column divided by one of nondoped columns in the simulated STEM image. The in-focus plane of the STEM probe is set to be on the entrance surface of the specimen when the defocus is zero. The amount of defocus indicates the deviation of the focus position from the surface. A bold arrow indicates the ratio of 1.3 . 